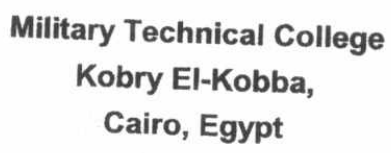

11-th International Conference on Aerospace Sciences \& Aviation Technology

\title{
NEW TECHNIQUE FOR SIMULATION OF FREQUENCY SELECTIVE SURFACES ( FSS )
}

\author{
M. H. Abdel-Azeem, Hossam Hamza and Ahmad Fawzy
}

\begin{abstract}
Two dimensional periodic arrays of patch elements can be used as frequency selective surfaces (FSS) owing to the frequency filtering properties of these structures. Arrays of square loops are of interest as frequency selective surfaces for dichroic reflector antennas. This paper describes a new way for modelling and analysis such FSS using the 3D Transmission Line Matrix method and compares the transmission characteristics predicted by the TLM method with those obtained experimentally.
\end{abstract}

\section{Methods of Simulation}

Frequency selective surfaces (FSS) are formed by a two dimensional array of metallic patterns printed on a dielectric substrate. The fundamental properties of FSS enable it to be used widely in many applications over the electromagnetic spectrum. In the microwave region, the frequency selective properties are largely exploited, for (1] and making efficient use of reflector antennas systems [2,3] where the FSS is used as the sub-reflector which enables two or more feeds to share the same parabolic main reflector simultaneously.

There are three methods which are currently used for analysis of FSS. The first method is a modal expansion analysis [4]. In this method, for infinite periodic arrays, the local fields scattered from the array elements are expanded as Floquet modes [4] is known as electromagnetic boundary conditions are applied. The second method s known as spectral iteration analysis [5]. The third method is to use an equivalent circuit model $[6,7]$. In the latter, the simplest method of analysis, the various strip segments that form a freestanding patch element in a periodic array are modeled as inductive and capacitive components on a transmission line. From the solution of this circuit, the reflection and transmission coefficients of the FSS are found. Since this approach uses the quasi-static approximation to calculate the circuit components, it is only accurate up to the resonant frequency of the FSS. In addition, it cannot model the dielectric loading effects accurately. Also, the expressions for the component
values are semi empirical.

The TLM is introduced in this paper as a new method for modelling the reflection and transmission coefficients of such FSS. TLM is a physical mode based on Huygen's 
principle using the equivalence between Maxwell's equations and the equations for voltage and currents on a mesh of continous two wire transmission lines (figure 1), that the equivalence is shown in its simplest form when we write the transmission line wave equation for the 2D-TLM, lossless homogenous case as:

$$
\frac{\partial^{2} V_{y}}{\partial x^{2}}+\frac{\partial^{2} V_{y}}{\partial z^{2}}=2 L C \frac{\partial^{2} V_{y}}{\partial t^{2}}
$$

and the equivalent Maxwell's equation is:

$$
\frac{\partial^{2} E_{y}}{\partial x^{2}}+\frac{\partial^{2} E_{y}}{\partial z^{2}}=\mu \varepsilon \frac{\partial E_{y}}{\partial t^{2}}
$$

that is, $V_{y} \equiv E_{y}, I_{z} \equiv H_{x}, I_{x} \equiv H_{z}, L \equiv \mu, 2 C \equiv$.

The analysis of the 2D-TLM can be made either using a shunt mesh or a series mesh while the widely used 3D-TLM SCN (Symmetrical Condensed Node) is formed using a combination of both (figure 2).

As described, voltage represent electric field in Maxwell's equation, thus electric wall is represented by short circuit while open circuit represents magnetic wall. This boundary is placed halfway between nodes to ensure synchronism. For examole, to set Ex equal to zero, all nodes Ex laying in this plane are shorted. Thus a reflection coefficient (1) represent open circuit \& (-1) for short circuit.

This equivalence allowed us to simulate the reflection and transmission characteristics for an incident TEM wave; an infinite surface would be necessary. So in this work we will use only one quarter of a single cell of the array placed in a TEM line consisting of two magnetic and two electric walls as shown in (figure 3). The perfectly reflecting walls act as mirrors to simulate the rest of the infinite structure.

The wave travelling in the line is TEM and the impedance of the line can be calculated exactly using an analytic formula. This means that the reference impedance, used in calculating the scattering parameters, is known exactly. The TEM line can be perfectly matched by terminating it with a plane with zero reflection coefficients. This means that both the TEM lines in front and behind the FSS need not to be very long since no reflections arise from the end of the line. These make the accurate and fast calculation of the FSS characteristics for a TEM wave possible.

The dielectric substrate in which the metallic pattern is printed could be very thin. In this case the dielectric layer is simulated only by an internal thin reflecting surface with the appropriate reflection coefficient.

Examples: A number of examples have been solved using the TLM simulator with no problems in stability. In all examples the above configuration was used to obtain the simulation results.

In the measurement method, the transmission characteristic of an FSS is obtained by illuminating it with a plane wave of different frequencies and measuring the loss caused by the FSS at the receiver side. This measurement provides a direct and rapid means of determining the performance of an FSS. 
Single FSS structures: Figure (4.a) shows the FSS structure to be simulated. The squares are all printed on the same surface of a thin dielectric substrate. Only one resonant frequency is expected.

Figure (4.b) shows a comparison between the measured and simulated results from either the TLM and the equivalent circuit model methods. Good agreements were obtained between the three sets of results.

Another array printed on a single surface but with two concentric squares is shown in (figure 5). The array was printed on a substrate $0.027 \mathrm{~mm}$ thin and with a dielectric constant of 3.0 .

Similar simulation and measurement procedures were followed and the results are compared in (figure 6).

Double layer frequency selective surfaces: To modify the transmission response of the FSS to match it to required operating bands, the double layer FSS shown in (figure 7) was investigated. The two layers are identical and they are separated by a dielectric medium $0.261 \mathrm{~mm}$ thick with $\epsilon_{\mathrm{r}}=3.78$.

This structure was designed for application in a diplexer where the two frequency bands (190-210 $\mathrm{GHz}$ and $540-660 \mathrm{GHz})$ were to be separated [8].

Figure (8) shows the results of the TLM simulation and (figure 9) shows the results of the measurements. Again in this case good agreement was obtained.

\section{Conclusion}

The use of TEM transmission lines and TLM offers a new method of simulating FSS with an incident TEM wave. The efficiency of the method results from the fact that only a quarter of a single cell need to be simulated and that short TEM lines can be used without any interfering reflections. The accuracy of the method results from the fact that the impedance of the TEM lines are known exactly. 


\section{References}

1. Pelton E. L. and Munk B. A., 1974, "A stream lined metallic radomes", IEEE Trans.Antennas and Propag., AP-22, pp. 799-803.

2. Agrawal V. D. and Imbriale W. A., 1979, "Design of a dichroic Cassegrian subreflector", IEEE Trans. Antennas and Propag., AP-27, pp. 466-473.

3. Comtesse L. E., Langley R. J., Parker E. A. and Vardaxoglou J. C., 1987, "Frequency selective surfaces in dual and triple band offset reflector antennas", Proc. 17 th European Microwave Conference, Rome, pp. 208-213

4. Amitay N., Galindo V. and Wu C. P., 1972, "Theory and analysis of phased array antennas", John Wiley \& Sons Inc.

5. Tsao C. H. and Mittra R., 1982, "A spectral iteration approach for analysing scattering from frequency selective surfaces", IEEE Trans. Antennas and Propag., Vol. AP-30, No. 2, pp. 303-308

6. Anderson I., 1975, "On the theory of self resonant grids", Bell Syst. Tech. J., 54, pp. $1725-1731$

7. Lee C. K. and Langley R. J., 1985, "Equivalent circuit models for frequency selective surfaces at oblique angles of incidence", IEE Proc. H, Vol. 132, pp. 395399.

8. Cahill R. and Parker E.A., 1999, "Frequency selective surface design for submillimetric demultiplexing", Microwave and optical Technology Letters. Vol. 7, No. 13, pp. 595-597. 

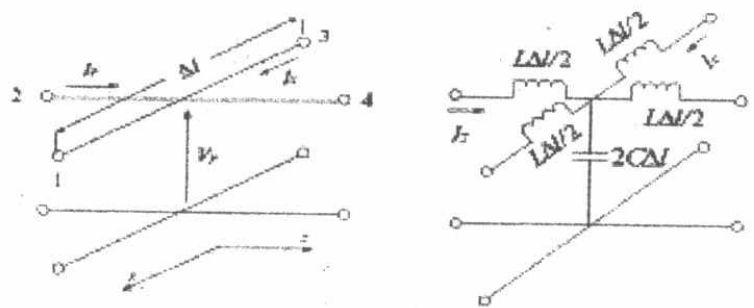

Figure 1: 2D-TLM shunt node and its transmission line equivalence circuit
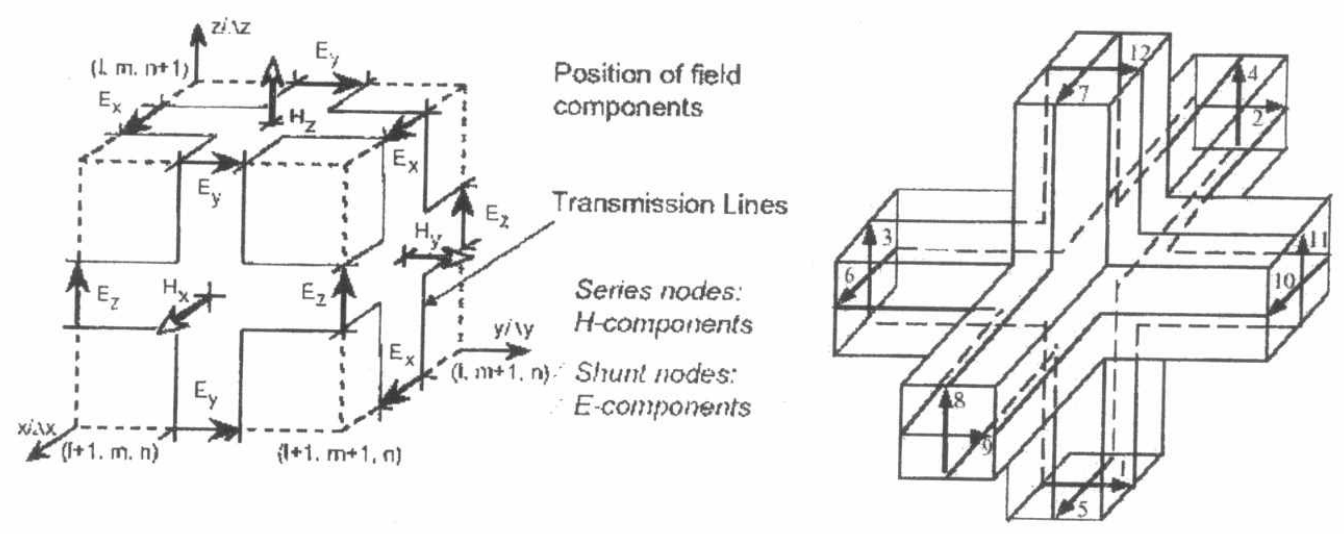

Figure 2a: 3D expanded TLM node consisting of $2 \mathrm{D}$ shunt and series nodes

Figure 2b: 3D-TLM SCN

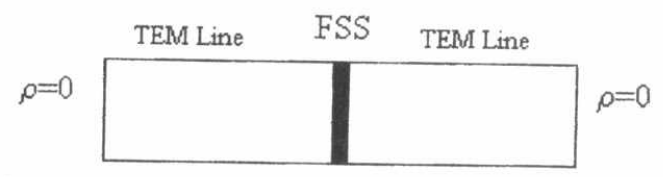

(a)

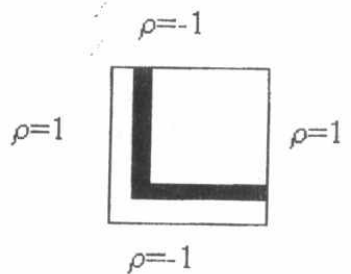

(b)

Figure 3: a) Simulated FSS in a TEM line

b) A quarter of a cell is used only 


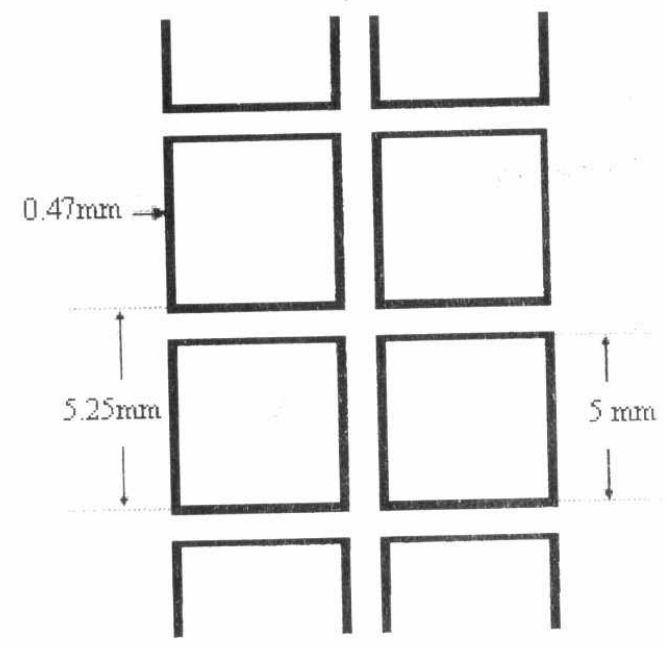

Figure 4a: A single square loop array loop array

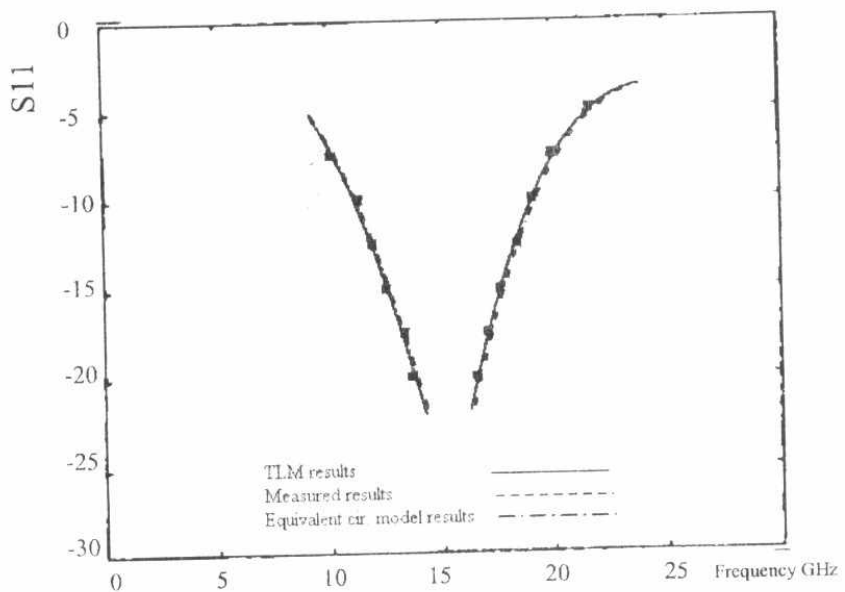

Figure 4b: Comparison between TLM, measured and Equivalent circuit model results for single 


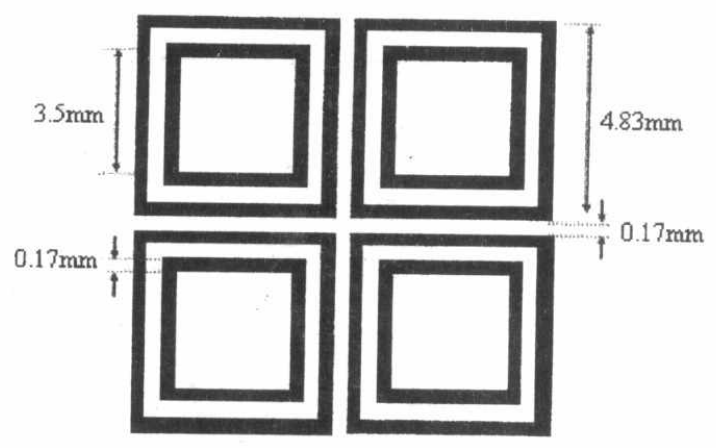

Figure 5: A Double square loop array

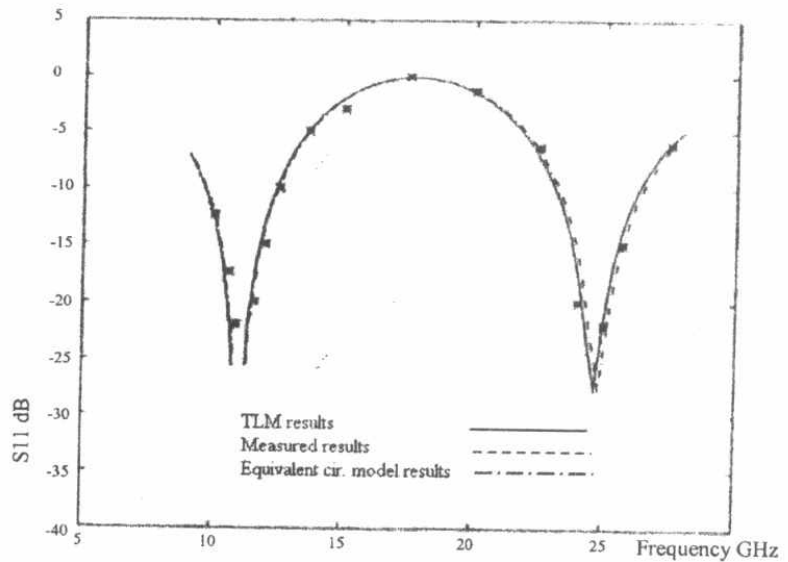

Figure 6: Comparison between TLM, measured and Equivalent circuit model results for double loop array

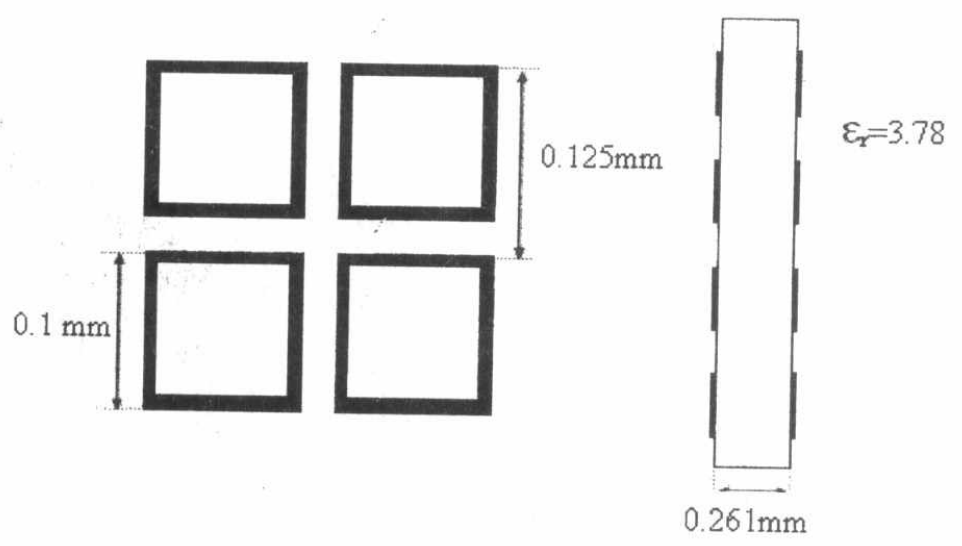

Figure 7: Printed square loop PCB-FSS 


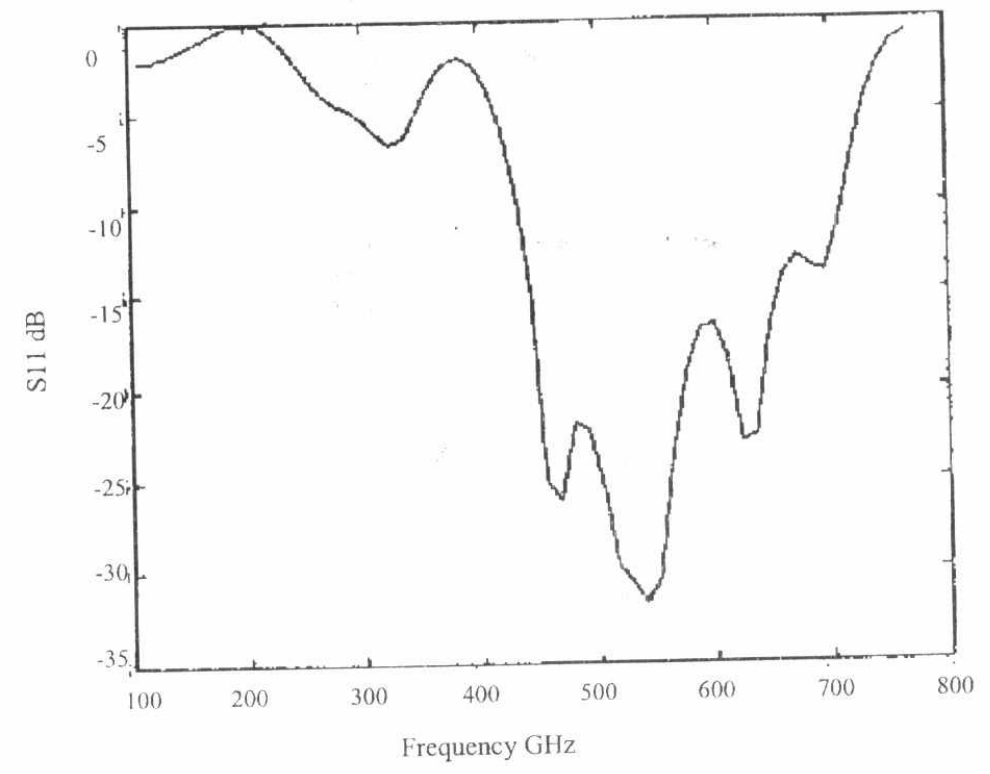

Figure 8: Transmission response of PCB-FSS from TLM results

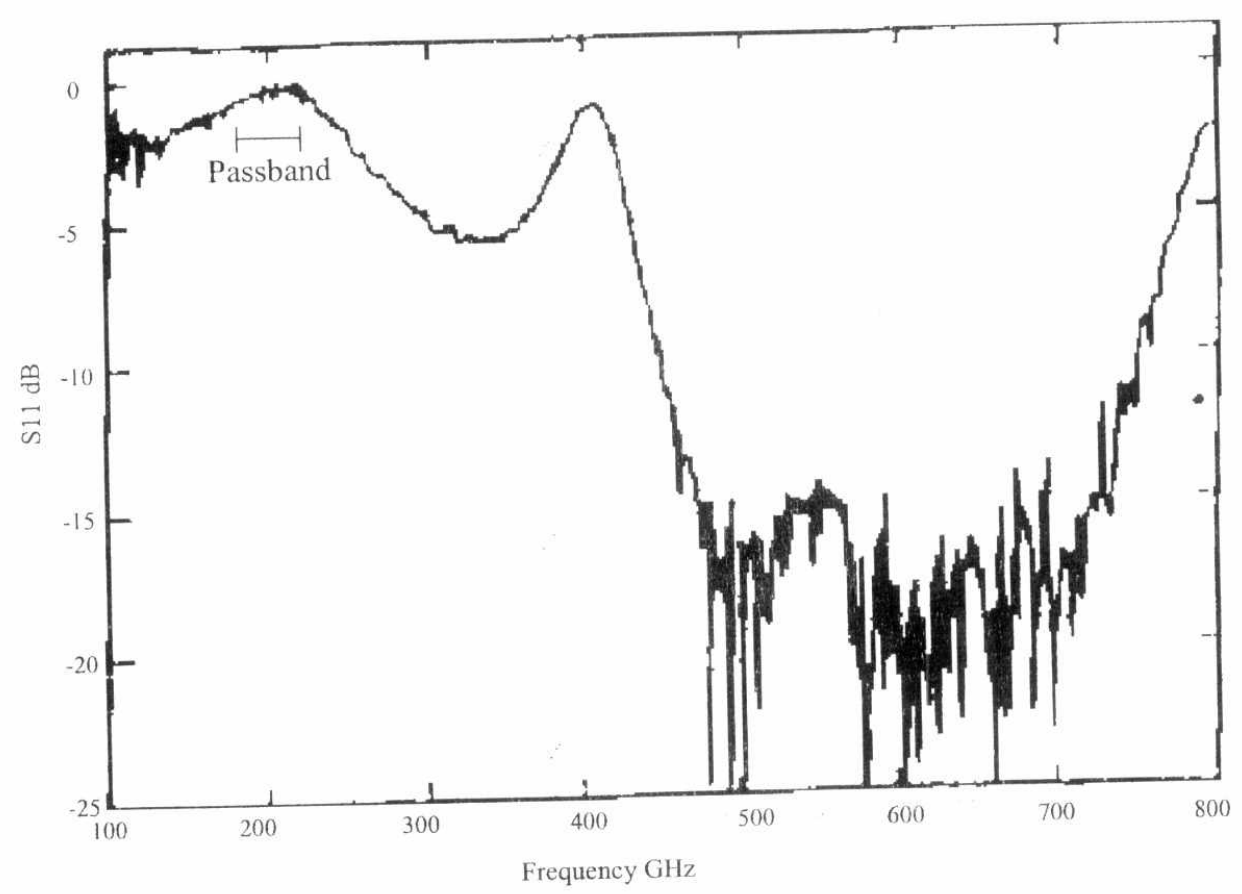

Figure 9: Transmission response of PCB-FSS from measurement results 